\title{
A simple approach to 3,6-branched galacto-oligosaccharides and its application to the syntheses of a tetrasaccharide and a hexasaccharide related to the arabinogalactans (AGs)
}

\author{
Jun Ning, ${ }^{\mathrm{a}, *}$ Hairong Wang ${ }^{\mathrm{b}}$ and Yuetao $\mathrm{Yi}^{\mathrm{a}}$ \\ ${ }^{a}$ Research Center for Eco-Environmental Sciences, Chinese Academy of Sciences, Beijing 100085, China \\ ${ }^{\mathrm{b}}$ Department of Chemistry, Tsinghua University, Beijing 100084, China
}

Received 20 June 2002; revised 9 August 2002; accepted 16 August 2002

\begin{abstract}
The preparation of 1,2:5,6-di- $O$-isopropylidene- $\alpha$-D-galactofuranose was improved by increasing the ratio of DMF to acetone and using a solid supported catalyst. Employing the easily accessible 1,2:5,6-di- $O$-isopropylidene- $\alpha$-D-galactofuranose as the starting glycosyl acceptor, a method which is particularly suitable for the regio- and stereoselective syntheses of 3,6-branched galacto-oligosaccharides was developed. A tetrasaccharide and a hexasaccharide related to the arabinogalactans (AGs) from plants were readily prepared using this strategy. (C) 2002 Elsevier Science Ltd. All rights reserved.
\end{abstract}

Increased appreciation of the role of carbohydrates in biological and pharmaceutical science has resulted in a revival of interest in carbohydrate chemistry. However, compared with other biopolymers such as peptides and nucleic acids, the role of the saccharide structure in function has been minimally studied. This can be attributed mainly to the difficulty of synthesizing saccharides. Therefore, the central problem in carbohydrate field is how to prepare oligosaccharides efficiently and simply. Although over the past few decades, considerable progress ${ }^{1}$ has been made in oligosaccharide synthesis, there is still a long way to go to find a general route for saccharide synthesis. Maybe, owing to this structural complexity, the preparation of saccharides will never achieve the same levels as the preparation of peptides and nucleic acids. But special methods which are suitable for a certain type of oligosaccharide synthesis can be developed.

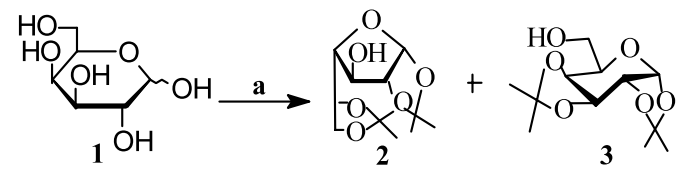

Scheme 1. Reagents and conditions: (a) DMF/acetone (4/1), Dry Hydrogen Resin, reflux, 15 h, 50\%.

\footnotetext{
* Corresponding author.
}

3,6-Branched D-Gal $p$ residues like $\beta$-D-galactopyranosyl- $(1 \rightarrow 6)-[\alpha-\mathrm{L}$-arabinofuranosyl- $(1 \rightarrow 3)]-\beta$ - D-galactopyranosyl and $3,6-\mathrm{di}-O$-( $\beta$-D-galactopyranosyl)- $\beta$-Dgalactopyranosyl are common structural components of arabinogalactans (AGs) from plants including traditional herbal medicines such as Lycium barbarum L., ${ }^{2}$ Bupleurum falcatum, ${ }^{3}$ Atractylodes lancea ${ }^{3}$ and Plantago major L. ${ }^{4}$ In addition to important roles in cell differentiation and development, they are involved in cell-cell interactions, elongation growth of the cell wall, and defense systems in plants. ${ }^{5}$ AGs possess various pharmacological activities. ${ }^{3}$ Yamada's research results show that AGs from A. lancea have intestinal immune system modulating activity in mice, and this effect is attributed to the $\beta-3,6$-D-galactan moiety in AGs. ${ }^{3}$ Although the presence of 3,6-branched $\beta$-D-Galp residues in AGs is well defined, the exact structure of these saccharides remains to be established. For detailed characterization of AGs, especially, for further elucidation of the molecular structure responsible for their biological activity, it would be necessary to synthesize 3,6-branched galactooligosaccharides. Reports on the synthesis of 3,6branched galacto-oligosaccharides are very limited. ${ }^{6}$ Here we disclose a special strategy for the synthesis of this kind of oligosaccharide, which involves an improved preparation of 1,2:5,6-di- $O$-isopropylidene- $\alpha$ D-galactofuranose and its use as the starting glycosyl acceptor. Syntheses of a tetrasaccharide and a hexasaccharide related to the AGs are presented as typical examples using the strategy developed. 
So far, 1,2:5,6-di- $O$-isopropylidene- $\alpha$-D-galactofuranose 2 has found only limited use in synthetic carbohydrate chemistry because it is not easily accessible. For example, 2 has been obtained from D-glucose derivatives in three or six steps, ${ }^{7}$ or from D-galactose in a maximum $22 \%$ yield. ${ }^{8}$ Recently, Rauter's group found that by using zeolite HY as the catalyst, the furanose diketal $\mathbf{2}$ was formed in $40 \%$ yield, together with the pyranose diketal 3, obtained only in $20 \%$ yield, and this significantly improved the preparation of $2 .{ }^{9}$ Some reports showed that high temperature is a factor known to favor furanose formation in solutions of reducing sugars. ${ }^{10}$ Inspired by this, we raised the ratio of DMF to acetone to 4 in our synthesis of 2, attempting to increase the reaction reflux temperature in order to obtain more furanose diketal $\mathbf{2}$. To simplify the purification procedure, an anhydrous $\mathrm{H}^{+}$ form cation exchange resin called Dry Hydrogen Resin (obtained from Nankai University, Ion Exchange and Adsorbent Resin Factory, Tianjin, China) was used as the solid supported catalyst (Scheme 1). As a result of our reaction conditions, the ratio of 1,2:5,6-di- $O$-isopropylidene- $\alpha$-D-galactofuranose to $1,2: 3,4$-di- $O$-isopropylidene- $\alpha$-D-galactopyranose in the reaction product can reach more than 4, and the desired 1,2:5,6-di- $O$-isopropylidene- $\alpha$-D-galactofuranose can be easily crystallized from the resultant residue in $50 \%$ yield.

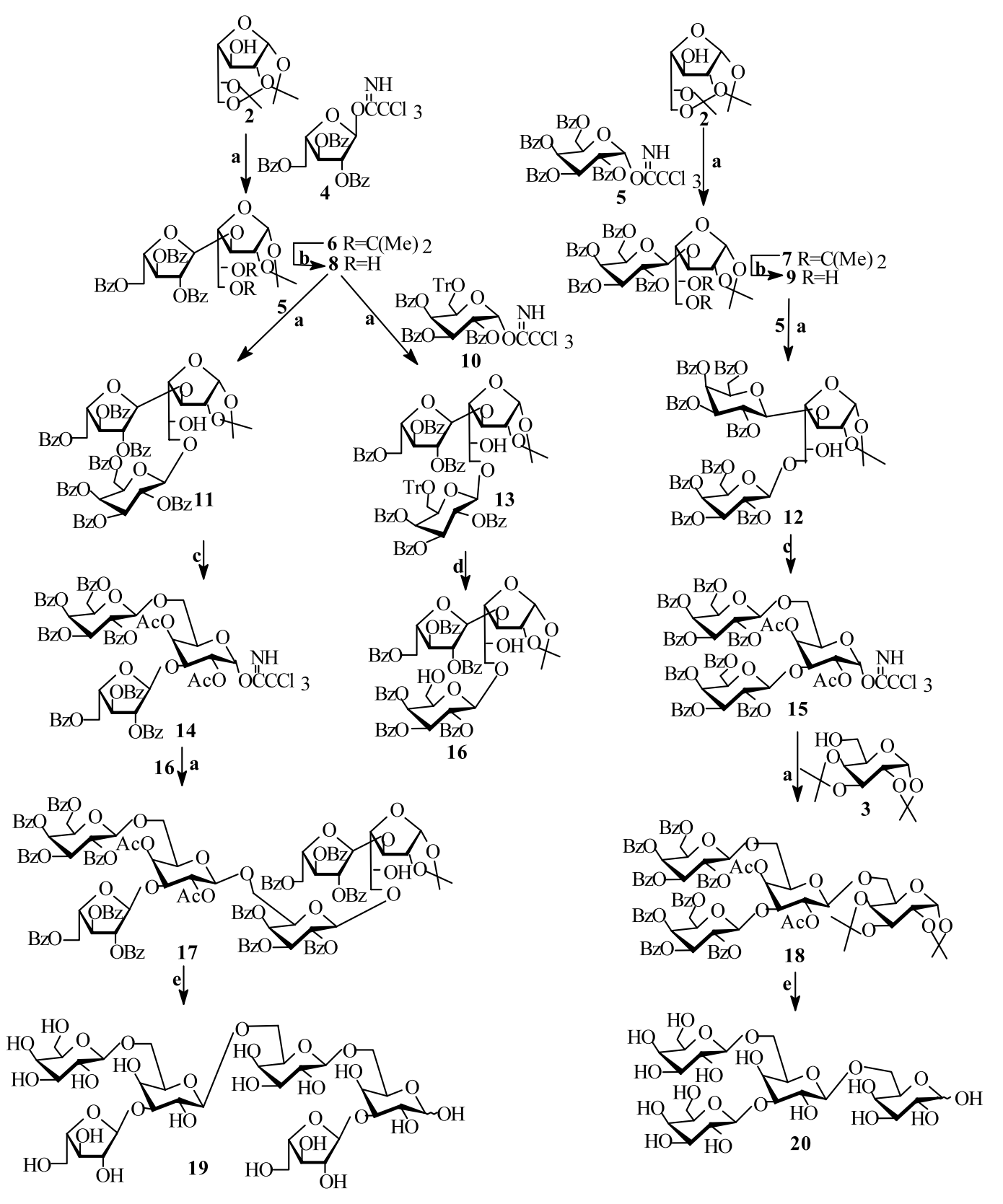

Scheme 2. Reagents and conditions: (a) TMSOTf (0.01 equiv.), $4 \AA \mathrm{AS}, \mathrm{CH}_{2} \mathrm{Cl}_{2}, \mathrm{rt}, 2-4$ h. ( $82 \%$ for $\mathbf{6}, 84 \%$ for 7, $85 \%$ for 11, $87 \%$ for $\mathbf{1 2}, 86 \%$ for $\mathbf{1 3}, 84 \%$ for 17, $91 \%$ for 18. (b) $90 \% \mathrm{AcOH}, 40^{\circ} \mathrm{C}, 2 \mathrm{~h}, 100 \%$. (c) (i) $80 \% \mathrm{AcOH}$, reflux, $3 \mathrm{~h}$; (ii) $\mathrm{Ac}_{2} \mathrm{O}$ /pyridine, rt, $10 \mathrm{~h}$; (iii) $\mathrm{THF} / \mathrm{CH}_{3} \mathrm{OH}, 1.5 \mathrm{~N} \mathrm{NH}_{3}, \mathrm{rt}, 2-3 \mathrm{~h}$; (iv) $\mathrm{CH}_{2} \mathrm{Cl}_{2}, \mathrm{CCl}_{3} \mathrm{CN}$ (2.0 equiv.), $\mathrm{K}_{2} \mathrm{CO}_{3}(2.0$ equiv.), rt, $12 \mathrm{~h}$, $71 \%$ for 14, $72 \%$ for 15 (for four steps). (d) $\mathrm{FeCl}_{3}$ (2 equiv.) in $\mathrm{CH}_{2} \mathrm{Cl}_{2}, \mathrm{rt}, 20 \mathrm{~min}, 90 \%$ for 16. (e) (i) $80 \% \mathrm{AcOH}$, reflux, $3 \mathrm{~h}$; (ii) $\mathrm{CH}_{2} \mathrm{Cl}_{2} / \mathrm{CH}_{3} \mathrm{OH}$ saturated with ammonia, rt, $36 \mathrm{~h}, 92 \%$ for $\mathbf{1 9}, 93 \%$ for 20 . 
Couplings of $\mathbf{2}$ with perbenzoyl arabinofuranosyl trichloroacetimidate $4^{6}$ and perbenzoyl galactopyranosyl trichloroacetimidate $\mathbf{5}^{11}$ in the presence of TMSOTf ( 0.01 equiv.) as catalyst, followed by selective 5,6- $O$-deacetonation afforded $\beta$ - $(1 \rightarrow 3)$-linked disaccharides 8 and $\mathbf{9}$, respectively, as solids in high yields (80-85\% for the two steps) (Scheme 2). Condensation of 5 with 8 and 9 catalyzed by TMSOTf regio- and stereoselectively gave the 3,6-branched trisaccharides $\mathbf{1 1}$ and 12, respectively, in excellent yields (85-90\%). Similarly, the $6^{\prime \prime}-O$-trityl trisaccharide $\mathbf{1 3}$ was obtained by coupling 2,3,4-tri- $O$-benzoyl-6- $O$-trityl- $\alpha$-D-galactopyranosyl trichloroacetimidate $\mathbf{1 0}^{12}$ with $\mathbf{8}$ in an excellent yield $(87 \%)$. Removal of the 1,2-O-isopropylidene group of 11 and 12 in $80 \%$ HOAc followed by acetylation with acetic anhydride in pyridine, selective 1-O deacetylation with ammonia in $\mathrm{THF} / \mathrm{CH}_{3} \mathrm{OH}$, and subsequent treatment with trichloroacetonitrile in the presence of $\mathrm{K}_{2} \mathrm{CO}_{3}$ afforded the trisaccharide glycosyl donors 14 and 15 in good yields $(71-74 \%$ over the four steps). Selective 6-O-detritylation of 13 in $\mathrm{CH}_{2} \mathrm{Cl}_{2}$ with $\mathrm{FeCl}_{3}$ gave the trisaccharide acceptor 16 in a high yield $(90 \%) .{ }^{13}$ Coupling of $\mathbf{1 4}$ with $\mathbf{1 6}$ using TMSOTf as the catalyst regio- and stereoselectively afforded the blocked hexasaccharide 17 in a high yield (84\%), while condensation of $\mathbf{1 5}$ with 1,2:3,4-di- $O$-isopropylidene- $\alpha$ D-galactopyranose 3 gave the blocked tetrasaccharide 18 in an excellent yield (91\%). ${ }^{14}$ Deisopropylidenation of $\mathbf{1 7}$ and $\mathbf{1 8}$ in $80 \%$ HOAc, followed by deacetylation in an ammonia-saturated solution in 1:1 $\mathrm{CH}_{2} \mathrm{Cl}_{2}$ / $\mathrm{CH}_{3} \mathrm{OH}$, furnished the free hexasaccharide 19 and tetrasaccharide 20, which are related to AGs, as amorphous white solids in $92 \%$ and $93 \%$ yields, respectively (for the two steps).

In all of the syntheses, easily accessible materials and cheap reagents were used and the reactions were carried out smoothly in high yields. Several intermediates were not separated but were used directly in further reactions thereby simplifying the procedures substantially.

In summary, a special strategy which is peculiarly suitable for the preparation of 3,6-branched galactooligosaccharides has been developed.

\section{Acknowledgements}

This work was supported by the Beijing Natural Science Foundation (6021004) and National Natural Science Foundation of China (59973026 and 29905004).

\section{References}

1. (a) Plante, O. J.; Palmacci, E. R.; Seeberger, P. H. Science 2001, 291, 1523; (b) Sears, P.; Wong, C. H. Science 2001, 291, 2344.

2. Peng, X.; Tian, G. Carbohydr. Res. 2001, 331, 95.

3. Yamada, H. In Bioactive Carbohydrate Polymers, Proceedings of the Phythochemical Society of Europe; Paulsen, B. S., Ed.; Kluwer Academic Publishers, 2000; Vol. 44, pp. 16-24.
4. Yamada, H. In Bioactive Carbohydrate Polymers, Proceedings of the Phythochemical Society of Europe; Paulsen, B. S., Ed.; Kluwer Academic Publishers, 2000; Vol. 44, pp. 37-46.

5. (a) Sussex, I. M. Cell 1989, 56, 225; (b) Kreuger, M.; van Host, G. J. Planta 1993, 189, 243; (c) Kreuger, M.; van Host, G. J. Planta 1994, 197, 135; (d) Egertsdotter, U.; Von Arnold, S. Physiol. Plant 1995, 93, 334; (e) Roberts, K. Curr. Opin. Cell. Biol. 1990, 2, 920; (f) Showalter, A. M.; Varner, J. E. In The Biochemistry of Plants; Stumpf, P. K.; Conn, E. E., Eds.; Academic: New York, 1989; Vol. 15, p. 485.

6. (a) Gu, G.; Yang, F.; Du, Y.; Kong, F. Carbohydr. Res. 2001, 336, 99; (b) Du, Y.; Pan, Q.; Kong, F. Carbohydr. Res. 2000, 323, 28.

7. (a) Jarosz, S.; Krajewski, J. W.; Zamojski, A.; Duddeck, H.; Kaiser, M. Bull. Pol. Acad. Sci. Chem. 1985, 33, 181; (b) De Jongh, D. C.; Biemann, K. J. Am. Chem. Soc. 1964, 86, 67.

8. (a) Morgenlie, S. Acta Chem. Scand. 1973, 27, 3609; (b) Morgenlie, S. Acta Chem. Scand. Ser. B 1975, 29, 367.

9. Rauter, A. P.; Ramoa-Riberio, F.; Fernanders, A. C.; Figueiredo, J. A. Tetrahedron 1995, 51, 6529.

10. (a) Acree, T. E.; Shallenberger, R. S.; Lee, C. Y.; Einset, J. W. Carbohydr. Res. 1969, 10, 355; (b) Acree, T. E.; Shallenberger, R. S.; Mattick, L. R. Carbohydr. Res. 1968, 6, 498.

11. Rio, S.; Beau, J. M.; Jacquinet, J. C. Carbohydr. Res. 1991, 219, 71.

12. Yu, B.; Xie, J.; Deng, S.; Hui, Y. J. Am. Chem. Soc. 1999, 121, 12196.

13. Ding, X.; Wang, W.; Kong, F. Carbohydr. Res. 1997, 303, 445.

14. All new compounds gave satisfactory elemental analysis results. Selected physical data for some key compounds are as follows: For 8: $[\alpha]_{\mathrm{D}}+46\left(c 2.1, \mathrm{CHCl}_{3}\right) .{ }^{1} \mathrm{H}$ NMR $\left(400 \mathrm{MHz}, \mathrm{CDCl}_{3}\right): \delta 5.96(\mathrm{~d}, 1 \mathrm{H}, J=4.0 \mathrm{~Hz}, \mathrm{H}-1), 5.59$ $\left(\mathrm{dd}, 1 \mathrm{H}, J=1.0,4.7 \mathrm{~Hz}, \mathrm{H}-3^{\prime}\right), 5.49(\mathrm{~d}, 1 \mathrm{H}, J=1.0 \mathrm{~Hz}$, H-2'), 5.45 (s, 1H, H-1'), 4.81 (dd, 1H, H-5a'), 4.74 (d, $1 \mathrm{H}, J=4.0 \mathrm{~Hz}, \mathrm{H}-2), 4.64\left(\mathrm{dd}, 1 \mathrm{H}, \mathrm{H}-5 \mathrm{~b}^{\prime}\right), 4.61(\mathrm{~m}, 1 \mathrm{H}$, H-4'), 4.40 (d, 1H, H-3), 4.18 (dd, 1H, H-4), $3.88(\mathrm{~m}, 1 \mathrm{H}$, $\mathrm{H}-5), 3.75$ (dd, 1H, H-6a), 3.68 (dd, 1H, H-6b), 1.54, 1.33 $\left(2 \mathrm{~s}, 6 \mathrm{H}, \mathrm{C}\left(\mathrm{CH}_{3}\right)_{2}\right)$; Anal. calcd for $\mathrm{C}_{35} \mathrm{H}_{36} \mathrm{O}_{13}: \mathrm{C}, 63.25$; H, 5.46. Found: C, 63.18; H, 5.51. For 9: $[\alpha]_{\mathrm{D}}+62(c 1.5$, $\left.\mathrm{CHCl}_{3}\right) .{ }^{1} \mathrm{H}$ NMR $\left(400 \mathrm{MHz}, \mathrm{CDCl}_{3}\right): \delta 5.99(\mathrm{~d}, 1 \mathrm{H}$, $\left.J=3.4 \mathrm{~Hz}, \mathrm{H}-4^{\prime}\right), 5.78\left(\mathrm{dd}, 1 \mathrm{H}, J=8.0,10.4 \mathrm{~Hz}, \mathrm{H}-2^{\prime}\right)$, $5.68(\mathrm{~d}, 1 \mathrm{H}, J=4.1 \mathrm{~Hz}, \mathrm{H}-1), 5.61(\mathrm{dd}, 1 \mathrm{H}, J=10.4,3.4$ $\left.\mathrm{Hz}, \mathrm{H}-3^{\prime}\right), 5.01\left(\mathrm{~d}, 1 \mathrm{H}, J=8.0 \mathrm{~Hz}, \mathrm{H}-1^{\prime}\right), 4.66(\mathrm{dd}, 1 \mathrm{H}$, H-6a'), 4.50-4.45 (m, 3H, H-6b', H-3, H-2), 4.37 (m, 1H, H-5'), 4.19 (dd, 1H, H-4), 3.89 (m, 1H, H-5), 3.70 (d, 2H, H-6a, 6b), 1.48, $1.24\left(2 \mathrm{~s}, 6 \mathrm{H}, \mathrm{C}\left(\mathrm{CH}_{3}\right)_{2}\right)$; Anal. calcd for $\mathrm{C}_{43} \mathrm{H}_{42} \mathrm{O}_{15}: \mathrm{C}, 64.66$; H, 5.30. Found: C, 64.75; H, 5.26. For 11: $[\alpha]_{\mathrm{D}}+28.1\left(c\right.$ 1.6, $\left.\mathrm{CHCl}_{3}\right) ;{ }^{1} \mathrm{H}$ NMR $(400 \mathrm{MHz}$, $\left.\mathrm{CDCl}_{3}\right): \delta 5.97(\mathrm{~d}, 1 \mathrm{H}, J=3.2 \mathrm{~Hz}, \mathrm{H}-4), 5.85(\mathrm{~d}, 1 \mathrm{H}$, $J=4.1 \mathrm{~Hz}, \mathrm{H}-1), 5.78$ (dd, $1 \mathrm{H}, \mathrm{H}-2), 5.61-5.58$ (m, $2 \mathrm{H}, 2$ $\mathrm{H}-3), 5.45$ (d, $1 \mathrm{H}, J=1.0 \mathrm{~Hz}, \mathrm{H}-2), 5.36$ (s, $1 \mathrm{H}, \mathrm{H}-1)$, $4.95(\mathrm{~d}, 1 \mathrm{H}, J=7.9 \mathrm{~Hz}, \mathrm{H}-1), 1.38,1.28(2 \mathrm{~s}, 6 \mathrm{H}$, $\left.\left(\mathrm{CCH}_{3}\right)_{2}\right)$; Anal. calcd for $\mathrm{C}_{69} \mathrm{H}_{62} \mathrm{O}_{22}: \mathrm{C}, 66.66 ; \mathrm{H}, 5.03$. Found: $\mathrm{C}, 66.97 ; \mathrm{H}, 5.00$. For 12: $[\alpha]_{\mathrm{D}}+34.9$ (c 2.3, $\left.\mathrm{CHCl}_{3}\right) ;{ }^{1} \mathrm{H}$ NMR $\left(400 \mathrm{MHz}, \mathrm{CDCl}_{3}\right): \delta 6.01(\mathrm{~m}, 2 \mathrm{H}, 2$ $\mathrm{H}-4), 5.83(\mathrm{dd}, 1 \mathrm{H}, \mathrm{H}-2), 5.78$ (dd, $1 \mathrm{H}, \mathrm{H}-2), 5.66$ (dd, $1 \mathrm{H}, \mathrm{H}-3), 5.62(\mathrm{dd}, 1 \mathrm{H}, \mathrm{H}-3), 5.55(\mathrm{~d}, 1 \mathrm{H}, J=4.1 \mathrm{~Hz}$, $\mathrm{H}-1), 4.50(\mathrm{~d}, 1 \mathrm{H}, J=8.0 \mathrm{~Hz}, \mathrm{H}-1), 4.96$ (d, 1H, $J=7.9$ 
$\mathrm{Hz}, \mathrm{H}-1), 1.28,1.14$ (2 s, 6H, $\left.\left(\mathrm{CCH}_{3}\right)_{2}\right)$; Anal. calcd for $\mathrm{C}_{77} \mathrm{H}_{68} \mathrm{O}_{24}$ : C, 67.15; H, 4.98. Found: C, 67.27; H, 5.03. For 13: $[\alpha]_{\mathrm{D}}+38.3\left(c 1.5, \mathrm{CHCl}_{3}\right) ;{ }^{1} \mathrm{H} \mathrm{NMR}(400 \mathrm{MHz}$, $\left.\mathrm{CDCl}_{3}\right): \delta 6.06(\mathrm{~d}, 1 \mathrm{H}, J=3.0 \mathrm{~Hz}, \mathrm{H}-4), 5.82(\mathrm{~d}, 1 \mathrm{H}$, $J=4.1 \mathrm{~Hz}, \mathrm{H}-1), 5.63-5.57$ (m, 3H, H-2, $2 \mathrm{H}-3), 5.42$ (d, $1 \mathrm{H}, J=1.4 \mathrm{~Hz}, \mathrm{H}-2), 5.34$ (s, 1H, H-1), $4.83(\mathrm{~d}, 1 \mathrm{H}$, $J=7.3 \mathrm{~Hz}, \mathrm{H}-1), 1.33,1.27$ (2 s, 6H, $\left.\left(\mathrm{CCH}_{3}\right)_{2}\right)$; Anal. calcd for $\mathrm{C}_{81} \mathrm{H}_{72} \mathrm{O}_{21}$ : C, 70.43; H, 5.25. Found: C, 70.31; $\mathrm{H}, 5.30$. For 14: $[\alpha]_{\mathrm{D}}+57.3\left(c 1.0, \mathrm{CHCl}_{3}\right) ;{ }^{1} \mathrm{H}$ NMR $(400$ $\left.\mathrm{MHz} \mathrm{CDCl}_{3}\right) \delta 8.34\left(\mathrm{~s}, 1 \mathrm{H}, \mathrm{CNHCCl}_{3}\right), 6.45(\mathrm{~d}, 1 \mathrm{H}$, $J=3.7, \mathrm{H}-1), 5.96(\mathrm{~d}, 1 \mathrm{H}, \mathrm{H}-4), 5.72(\mathrm{dd}, 1 \mathrm{H}, \mathrm{H}-2), 5.64$ (dd, 1H, H-3), 5.53 (dd, 1H, H-3), 5.40 (d, 1H, J=1.2 Hz, H-2), 5.39 (s, 1H, H-1), 5.31 (dd, 1H, H-2), 4.93 (d, $1 \mathrm{H}, J=7.7 \mathrm{~Hz}, \mathrm{H}-1), 2.08,1.86\left(2 \mathrm{~s}, 6 \mathrm{H}, 2 \mathrm{CH}_{3} \mathrm{CO}\right) ;{ }^{13} \mathrm{C}$ NMR (100 MHz, $\left.\mathrm{CDCl}_{3}\right): \delta 169.9,169.1\left(2 \mathrm{CH}_{3} \mathrm{CO}\right)$, $160.4\left(\mathrm{OC}(\mathrm{NH}) \mathrm{CCl}_{3}\right), 107.0,100.3,93.4$ (3 C-1), 90.4 $\left(\mathrm{OC}(\mathrm{NH}) \mathrm{CCl}_{3}\right), 20.0,19.9\left(2 \mathrm{CH}_{3} \mathrm{CO}\right)$. Anal. calcd for $\mathrm{C}_{72} \mathrm{H}_{62} \mathrm{Cl}_{3} \mathrm{NO}_{24}$ : C, 60.41; H, 4.36. Found: C, 60.19; $\mathrm{H}$, 4.31. For 15: $[\alpha]_{\mathrm{D}}+21.8\left(c 1.0, \mathrm{CHCl}_{3}\right) ;{ }^{1} \mathrm{H}$ NMR $(400$ $\left.\mathrm{MHz}, \mathrm{CDCl}_{3}\right) \delta 8.23\left(\mathrm{~s}, 1 \mathrm{H}, \mathrm{CNHCCl}_{3}\right), 6.33(\mathrm{~d}, 1 \mathrm{H}$, $J=3.7, \mathrm{H}-1), 5.97$ (d, 1H, H-4), 5.93 (d, 1H, H-4), 5.75 (dd, 1H, H-2), 5.68 (dd, 1H, H-2), $5.56(\mathrm{dd}, 1 \mathrm{H}, \mathrm{H}-3)$, $5.54(\mathrm{dd}, 1 \mathrm{H}, \mathrm{H}-3), 5.16(\mathrm{dd}, 1 \mathrm{H}, \mathrm{H}-2), 4.95(\mathrm{~d}, 1 \mathrm{H}$, $J=7.7 \mathrm{~Hz}, \mathrm{H}-1), 4.92$ (d, 1H, $J=8.0 \mathrm{~Hz}, \mathrm{H}-1), 2.17,1.46$ (2 s, $6 \mathrm{H}, 2 \mathrm{CH}_{3} \mathrm{CO}$ ); Anal. calcd for $\mathrm{C}_{80} \mathrm{H}_{68} \mathrm{Cl}_{3} \mathrm{NO}_{26}: \mathrm{C}$, 61.37; H, 4.38. Found: C, 61.49; H, 4.31. For 16: $[\alpha]_{\mathrm{D}}$ $+51.9\left(c\right.$ 1.2, $\left.\mathrm{CHCl}_{3}\right) ;{ }^{1} \mathrm{H} \mathrm{NMR}\left(400 \mathrm{MHz}, \mathrm{CDCl}_{3}\right): \delta$ $5.90(\mathrm{~d}, 1 \mathrm{H}, J=4.1 \mathrm{~Hz}, \mathrm{H}-1), 5.81$ (dd, 1H, H-2), 5.77 (d, $1 \mathrm{H}, J=3.0 \mathrm{~Hz}, \mathrm{H}-4), 5.60(\mathrm{dd}, 1 \mathrm{H}, \mathrm{H}-3), 5.54(\mathrm{dd}, 1 \mathrm{H}$, $\mathrm{H}-2), 5.47$ (d, 1H, J=1.4 Hz, H-2), 5.40 (s, 1H, H-1),
4.87 (d, 1H, J=7.6 Hz, H-1), 1.39, 1.29 (2 s, 6H, $\left.\left(\mathrm{CCH}_{3}\right)_{2}\right) ;{ }^{13} \mathrm{C} \mathrm{NMR}\left(100 \mathrm{MHz}, \mathrm{CDCl}_{3}\right): \delta 166.1,165.7$,

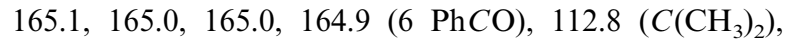
104.9, 104.7, 101.6 (3C-1), 26.4, $\left.25.9\left(\mathrm{C}_{(\mathrm{CH}}\right)_{2}\right)$. Anal. calcd for $\mathrm{C}_{62} \mathrm{H}_{58} \mathrm{O}_{21}$ : C, 65.37; H, 5.13. Found: C, 65.27; $\mathrm{H}$, 5.08. For 17: $\left.[\alpha]_{\mathrm{D}}+55.3(c) 3.6, \mathrm{CHCl}_{3}\right) ;{ }^{13} \mathrm{C} \mathrm{NMR}$ $\left(100 \mathrm{MHz}, \mathrm{CDCl}_{3}\right): \delta 169.2,169.1\left(2 \mathrm{CH}_{3} \mathrm{CO}\right), 113.0$ $\left(C\left(\mathrm{CH}_{3}\right)_{2}\right), 107.2,104.7,104.5,101.0,100.7,100.5$ (6C-1), 26.5, $26.0\left(2 \mathrm{C}\left(\mathrm{CH}_{3}\right)_{2}\right), 20.1,18.7\left(2 \mathrm{CH}_{3} \mathrm{CO}\right)$. Anal. calcd for $\mathrm{C}_{132} \mathrm{H}_{118} \mathrm{O}_{44}$ : C, 65.83; H, 4.94. Found: $\mathrm{C}, 65.39 ; \mathrm{H}$, 5.02. For 18: $[\alpha]_{\mathrm{D}}+78.8\left(c 1.8, \mathrm{CHCl}_{3}\right) ;{ }^{1} \mathrm{H}$ NMR $(400$ $\left.\mathrm{MHz}, \mathrm{CDCl}_{3}\right): \delta 5.99(\mathrm{~d}, 1 \mathrm{H}, \mathrm{H}-4), 5.92(\mathrm{~d}, 1 \mathrm{H}, \mathrm{H}-4)$, 5.81 (dd, 1H, H-2), 5.68 (dd, 1H, H-2), 5.58-5.54 (m, 2H, $2 \mathrm{H}-3), 5.46$ (d, 1H, J=4.9 Hz, H-1), 5.12 (dd, 1H, H-2), $4.98(\mathrm{~d}, 1 \mathrm{H}, J=8.0 \mathrm{~Hz}, \mathrm{H}-1), 4.86(\mathrm{~d}, 1 \mathrm{H}, J=7.7 \mathrm{~Hz}$, $\mathrm{H}-1), 4.25$ (d, 1H, J=7.9 Hz, H-1), 2.18, 1.57 (2 s, 6H, 2 $\left.\mathrm{CH}_{3} \mathrm{CO}\right), 1.42,1.35,1.29,1.28\left(4 \mathrm{~s}, 12 \mathrm{H}, 2\left(\mathrm{CCH}_{3}\right)_{2}\right) ;{ }^{13} \mathrm{C}$ NMR (100 MHz, $\left.\mathrm{CDCl}_{3}\right): \delta 169.3,168.2\left(2 \mathrm{CH}_{3} \mathrm{CO}\right)$, 108.9, $108.0\left(2 \mathrm{C}\left(\mathrm{CH}_{3}\right)_{2}\right), 101.6,101.0,100.9,95.8$ (4C-1), 25.6, 25.4, 24.6, $23.7\left(2 \mathrm{C}\left(\mathrm{CH}_{3}\right)_{2}\right), 20.2,19.7\left(2 \mathrm{CH}_{3} \mathrm{CO}\right)$. Anal. calcd for $\mathrm{C}_{90} \mathrm{H}_{86} \mathrm{O}_{31}$ : C, 64.98; H, 5.21. Found: $\mathrm{C}$, 65.16; H, 5.28. For 19: $[\alpha]_{\mathrm{D}}-9.6\left(c\right.$ 2.0, $\left.\mathrm{H}_{2} \mathrm{O}\right) ;{ }^{13} \mathrm{C} \mathrm{NMR}$ (100 MHz, D 2 ): 109.73, 109.61, 104.37, 104.26, 103.97 $\left(\mathrm{C}-1_{\mathrm{B}}, 1_{\mathrm{C}}, 1_{\mathrm{D}}, 1_{\mathrm{E}}, 1_{\mathrm{F}}\right), 98.1\left(\mathrm{C}-1_{\mathrm{A}}\right.$ for $\left.\beta\right), 94.3\left(\mathrm{C}-1_{\mathrm{A}}\right.$ for $\left.\alpha\right)$. ES MS. calcd for $\mathrm{C}_{34} \mathrm{H}_{58} \mathrm{O}_{29}$ : 930.81 [M]. Found: 953.8 $(M+\mathrm{Na})^{+}$. For 20: $\left.[\alpha]_{\mathrm{D}}-19.2(c) 1.6, \mathrm{H}_{2} \mathrm{O}\right) ;{ }^{13} \mathrm{C} \mathrm{NMR}$ $\left(100 \mathrm{MHz}, \mathrm{D}_{2} \mathrm{O}\right)$ : 104.0, 103.8, $102.86\left(\mathrm{C}-1_{\mathrm{B}}, 1_{\mathrm{C}}, 1_{\mathrm{D}}\right)$, $97.25\left(\mathrm{C}-1_{\mathrm{A}}\right.$ for $\left.\beta\right), 93.09\left(\mathrm{C}-1_{\mathrm{A}}\right.$ for $\left.\alpha\right)$. ES MS. calcd for $\mathrm{C}_{24} \mathrm{H}_{42} \mathrm{O}_{21}: 666.58[M]$. Found: $689.6(M+\mathrm{Na})^{+}$. 